\title{
Intellectual Function among Epileptic Children: The Role of Epilepsy Related-Factors in Nigeria
}

\author{
Koleoso $\mathrm{ON}^{1} \& U$ wadiae $\mathrm{E}^{2}$ \\ ${ }^{1,2}$ (Department of Mental Health, University of Benin Teaching Hospital, Benin City, Nigeria)
}

\begin{abstract}
This study evaluated the role of epilepsy-related factors, such as etiological variability, sex and age, on general intellectual functions among Nigerian children with epilepsy. Sixty epileptic children (38 males and 22 females) with a mean age of 12.9 years ( $S D=2.9$ years) were randomly selected from a population of epileptic children attending the Outpatient Neurology Clinic at Neuropsychiatric Hospital, Aro, Abeokuta. The Wechsler Intelligence Scale for Children (WISC-R) was administered. The result showed that $73.4 \%$ of the epileptic children had mental retardation, borderline and low average IQ level which, in some way, corresponded to general intellectual impairment. Multiple regression analysis revealed that etiological variability independently predicted Verbal IQ, Performance IQ and Full Scale IQ among the epileptic children. Furthermore, sex independently predicted Performance IQ of the epileptic children. This study emphasised a significant joint prediction of etiological variability, sex and age on Verbal IQ, Performance IQ and Full Scale $I Q$ among the epileptic children. In conclusions, etiological variability and sex are important predictors of general intellectual functions. This research will enhance the understanding of those epileptic children who are in danger of developmental impairment so that treatment methods can be developed.
\end{abstract}

Keywords: Epileptic children, Full IQ, General intellectual functions, Performance IQ, Verbal IQ

\section{Introduction}

Epilepsy is acondition caused by neurological deficit and is one of the frequent neurological disorders in childhood[1]. Epilepsy is defined as a chronic state distinguished by a predisposition towards recurrent seizures[2]. It is a regular disorder resulting from seizures that momentarily damage brain function. The incidence of cognitive impairments is an unavoidable aspect of epileptic practice. At the same time, neurologists, psychiatrists, and clinical psychologists treating epilepsy in children often underestimate these impairments. Paying no heed to patient's cognitive deficits at the early phase of sickness can afterward lead to worsening of these crises, which hampers management. For moments, on the contrary, there is overvaluation of cognitive impairments in patients with epilepsy, when virtually any of a patient's behavioural or personality characteristics can be linked to the surroundings or with the disease [3].Pervasiveness estimates propose that approximately $5 \%$ of children will have at least one seizure in their lifetime with approximately $25 \%$ of these children consequently meeting formal diagnostic criteria for epilepsy $[4,5]$. Neurocognitively, these children have been shown to be at danger for a diversity of neuropsychological deficits as well as deficits in, or the whole intellectual functioning, memory, attention, and executive functioning [6].

Elger, Helmstaedter and Kurthen[7] claim that neuropsychological impairment is an essential comorbidity of chronic epilepsy. Research has described associations between cognitive standing and a range of clinical epilepsy features, including etiology, age of onset, seizure type and severity, duration, antiepileptic medications, and other factors $[8,9]$. Cognitive and neuropsychological dysfunctions are common in children with epilepsy. Epilepsy in children presents most important management challenges for it is commonly linked with learning and behavioural problems, ever-increasing the risk of educational underachievement [10].

Recently, cognitive outcome of epilepsy has been given a great deal of attention because cognitive deficits can have harmful effects on every area of a person's life, such as academic performance, occupation, interpersonal relations and even leisure activities [11]. In children, it can significantly impair the learning process, obstructing the child's prospect competences. Adjustment at school can be affected owing to behaviour problems consequent to cognitive dysfunction for example,the distracted epileptic child may be scolded, leading to maladaptive behaviour at school which can be extended to other situations [12].

At the moment, it is commonly admitted that a division of people with epilepsy will experience a number and varying degree of cognitive impairment. An association between childhood epilepsy and cognitive dysfunction has long been identified [13]. Cognitive impairments have been linked to communications among genetics, ongoing seizures, different epileptic syndromes, subclinical epileptiform discharges, psychosocial issues, underlying causes of symptomatic epilepsy, and treatment with antiepileptic drugs (AEDs) [14]. According to You[13], all of these factors are interconnected, and their contributions to cognitive deficits are 
complex. Predictors of cognitive deterioration in these patients include overmedication, poor seizure control, and young age at onset of epilepsy [15].

Etiologically, there are two groups of epilepsy. The 'idiopathic' or 'uncomplicated' epilepsy, where no cause can be established; and 'symptomatic' epilepsyassociated with organic pathology or complicated by other neurological difficulties. According to van Rijckevorsel[16], etiology is an especially significant issue. Idiopathic epilepsy patients grow healthier than symptomatic cases. Nevertheless, this common contention suffers from a number of exceptions. For example, Dravet syndrome is a genetic epileptic syndrome with a cruel prognosis, whereas some cryptogenic focal epilepsy may well have a promising development with a rapid and absolute seizure control and no cognitive deficits. Bhise et al. [14] reported cognitive impairments, mostly in attention tasks, in a group of children with idiopathic epilepsy, regardless of seizure type. Berg et al. [17] showed that impaired cognitive performance in children with symptomatic focal seizures is more explicit and directly related to the most affected brain region.

Children with symptomatic epilepsy are at a bigger danger for cognitive impairment than are those with idiopathic epilepsy [15]. Takagi [18] evaluated intellectual functions of epileptic children using the Wechsler Intelligence Scale for Children (WISC). He found that both the Verbal IQ and Performance IQ were significantly lower in the symptomatic group than in the idiopathic group. Studies of patients with idiopathic epilepsies may be valuable in appreciating whether cognitive impairment is related to steady, disease-related attributes of the epilepsy or to paroxysmal epileptic activity, as well as the severe effects of seizures and the results of interictalepileptiform discharges as monitored on electroencephalogram (EEG)[19].

Additionally, developmental difficulties are more frequent in children with symptomatic epilepsy than in children with idiopathic epilepsy $[20,21]$. In the majority idiopathic straightforward epilepsies, cognition is slightly impaired or within standard domain [22]. Normal development and average intellectual functioning are linked with idiopathic epilepsies [23]. In spite of the benign medical prognosis, a range of neurocognitive deficits has been established in children with idiopathic epilepsies. Aldenkamp, Overweg-Plandson, \&Diepman[24] claim that mental-slowing impairments in attention and memory are quite classic in children with epilepsy. Henkin et al. [25] have reported specific deficits in visual and verbal attention problem among the school-age children with idiopathic epilepsy. Deonna, Zesiger, Davidoff, Maeder, and Roulet[26] aver that the inconsistency of results make obvious that there exists no single pattern of neuropsychological impairment in idiopathic epilepsy. As an alternative, a more diffuse neuropsychological profile with benign childhood epilepsy has been suggested [27].

Guzeva, Belash, Guzeva, Guzeva, \&Anastazi[28], while exploring the characteristic of cognitive functions in children with epilepsy, note that increased intensity of active attention and mental capacity were encountered with essentially corresponding regularities in symptomatic generalised and idiopathic epilepsy, despite the fact that these symptoms were less recurrent in patients with symptomatic partial epilepsy. Decreased memory was observed in the entire types of epilepsy, with an insignificant high proportion of reduced short-term memory in symptomatic generalised and long-term memory in symptomatic partial epilepsy. Impairment to intellectual functions was noted in patients with different types of epilepsy, but further considerable adjustments were observed in idiopathic epilepsy.

Sharma et al. [11] assert that male epileptic children had more neurological deficits than females but female epileptic children experience more psychological deficits. It has also been argued that gender had no impact on neurological and psychological deficits among epileptic children. Other similar results show that gender is inconsistent as a predictor of psychological problems in children with epilepsy. Hoare and Kerley[29] found no gender difference but Austin et al. [30] found that boys had more problems than girls, whereas a previous study of children with chronic epilepsy by Austin et al. showed that girls with more severe seizures had the most difficulties.

Sharma et al. [11], in a descriptive study to evaluate the neuropsychological deficits among epileptic children,found that both neurological deficits and psychological deficits were uppermost in the age group of 1316 years. Sridharan and Murthy [31] also found that age-specific frequency rates were higher in the younger age group, with the onset of epilepsy reported regularly in the first three decades of the sample population's lives. The study concluded that the majority of the epileptic children with neurological deficits had age at onset of seizures at 11-13 years but psychological deficits were more among epileptic children who were having age at onset of seizure before the age of four.

Determination of general intellectual functions among epileptic children in Nigeria using standardized measure of intelligence has been largely unexplored and unknown. This research will enhance understanding of the problem in this population so that treatment methods can be developed. The objectives of this study were to determine the percentage distribution of the general intellectual functions and also to establish which epilepsiesrelated factors would predict general intellectual functions of epileptic children. Determining predictors of poor result is significant in recognizing those mainly at danger for developmental impairment. Therefore, based on 
previous research, we hypothesised that etiological variability, sex and age would independently and jointly predict Verbal IQ, Performance IQ and Full Scale IQ of epileptic children.

\section{Materials And Methods}

\subsection{Design of the study}

This study adopted a cross-sectional survey design, utilized to assess the general intellectual function among epileptic children. The independent variables were etiological variability (symptomatic and idiopathic) sex (male and female) and age (6-12 years and $13-16$ years). The dependent variable was general intellectual functioning, which included Verbal IQ, Performance IQ and Full Scale IQ.

\subsection{Setting}

This study was conducted at the Outpatient Clinic of Neuropsychiatric Hospital, Aro, Abeokuta, Ogun State, Nigeria. Selection of field was done because of reasons such as familiarity with the setting, availability of subjects, administrative approval, and economy of time, money, and energy.

\subsection{Participants}

The study participants were selected through the purposive sampling technique at the Outpatient Clinic. Of the 60 patients eligible for the study, $38(63.3 \%)$ were male, while $22(36 \%)$ were female. In terms of etiological variability, symptomatic epilepsy was identified in $32(53.3 \%)$ children, while idiopathic epilepsy was identified in $28(46.7 \%)$ children. The age of participants between 6 and 12 was $30(50.0 \%)$; age group 13 and 16 was also $30(50.0 \%)$. The team identified $42(70 \%)$ children with generalised seizures, and the remaining $18(30 \%)$ with unclassified seizure types. More than half of the children $(53.3 \%)$ were receiving monotherapy of an anti-epileptic drug for seizure control.

\subsection{Procedure}

Epileptic children without any gross mental abnormality or other diagnosable neurological disorders, between the ages 6 and16 years, on the basis of informed consent obtained from their parents and the willingness of the children to participate in the study, were taken for the study. All the children had a single neuropsychological assessment of about two hours after a routine medical examination at the Outpatient Clinic of Neuropsychiatric Hospital, Aro, Abeokuta, Ogun State. The detailed medical and psychiatric history was done by a senior registrar at the clinic who also determined the children's' etiological variability classification in terms of symptomatic or idiopathic epilepsy. In this study, symptomatic epilepsy is taken to mean epilepsy that can be traced to a known cause, such as having either jaundice in the neonatal period, febrile convulsions in childhood, head injury in childhood and/or severe systemic infection in childhood, while idiopathic epilepsy is epilepsy that cannot be traced to any known cause. All assessments were made by the same experienced Clinical psychologist. The Intellectual Coefficient (IQ) was calculated from the application of the Wechsler Intelligence Scale for Children - Revised (WISC-R), a scale of standardized intelligence tests, adapted for Nigerian children aged 6 to16 years and 11 months. IQ is divided into Verbal IQ and Performance IQ and comprises a total of 12 subtests. Verbal IQ evaluates one's ability to express and receive verbal and abstract reasoning, and is culturally biased. The Verbal IQ test is made up of six subtests: Information, Similarities, Arithmetic, Vocabulary, comprehension and Digit span. The Performance IQ assesses capacity planning, integration and perceptual processing speed, and most activities require measuring the time spent to complete them. Performance IQ consists of seven subtests: Picture completion, Coding, Picture arrangement, Block design, Object assembly, Symbol search (additional) and Mazes (additional). The score is found by obtaining the raw score of each subtest and converting it into a score according to the age of the child; this score is, in turn, converted into an IQ value. Level of intelligence is classified as superior (IQ score $\geq 120$ ), high average (IQ score of 110-119), average (IQ scores of 90-109), low average (IQ scores of 80-89), borderline (IQ scores of 70-79) and mentally challenged (IQ $\leq 69)$, according to the rules of the test manual.

\subsection{Data analysis}

All statistical analysis was performed using the Statistical Package for the Social Sciences (SPSS) (version 15.0). For the comparison of the intellectual functioning of the study, Verbal IQ, Performance IQ and Full Scale IQ were calculated. Standard scores and z- scores for age groups were used in the analysis of the group differences. Apart from descriptive statistics, the analyses includedcalculation of multiple regression analyses to assess the joint influence of the independent variables on intellectual functioning. The results were considered statistically significant when $\mathrm{p}<0.05$. 


\section{Results}

The measurement of general intellectual functions among epileptic children revealed that $73.4 \%$ of epileptic children had mental retardation, borderline and low average IQ level which, in some way, corresponded to general intellectual impairment. Hence, it can be concluded that epilepsy had some impact on the general intellectual functioning of the children. In the present study, $36.7 \%$ of the epileptic children actually were mentally retarded (Table 1$)$.

Table 1: Percentage Distribution of General Intellectual Functions(GIF) AmongChildren (EC) According to Level of IQ

\begin{tabular}{|l|l|l|}
\hline Classifications IQ & \multicolumn{2}{l|}{ GIFs among EC } \\
\hline & N & $\%$ \\
\hline Superior (<120) & - & - \\
\hline High Average (110-119) & 2 & 3.3 \\
\hline Average (90-109) & 14 & 23.3 \\
\hline Low Average (80-89) & 13 & 21.7 \\
\hline Borderline (70-79) & 19 & 15.0 \\
\hline Mental Retardation (Below 70) & 22 & 36.7 \\
\hline Total & 60 & 100 \\
\hline
\end{tabular}

Regarding the determinants of general intellectual functions among children with epilepsy, multiple regression analysis was used to test the independent and joint variables that would yield an optional predictive equation of Verbal Intelligence Quotient (Verbal IQ) among the epileptic children. The independent variables selected were etiological variability,sex and age. The results are presented below:

Table 2: Table Showing the MultipleRegression Analysis on Predictive Ability of Sex, Age and Etiology on Verbal IQ, Performance IQ and Full IQ

\begin{tabular}{|c|c|c|c|c|c|c|c|c|}
\hline & Variables & B & $\beta$ & $\mathbf{T}$ & $\mathbf{P}$ & $\mathbf{R}^{2}$ & $\mathbf{F}$ & $\mathbf{P}$ \\
\hline \multirow[t]{3}{*}{ Verbal IQ } & Sex & -5.66 & -.15 & -1.18 & $>.05$ & \multirow[t]{3}{*}{.19} & \multirow[t]{3}{*}{4.27} & \multirow[t]{3}{*}{.01} \\
\hline & Age & .09 & .00 & .02 & $>.05$ & & & \\
\hline & Etiology & 13.42 & .37 & 2.99 & $<.01$ & & & \\
\hline \multirow{3}{*}{$\begin{array}{l}\text { Performance } \\
\text { IQ }\end{array}$} & Sex & -14.02 & -.27 & -2.13 & $<.05$ & \multirow[t]{3}{*}{.22} & \multirow[t]{3}{*}{5.30} & \multirow[t]{3}{*}{.01} \\
\hline & Age & 34 & .00 & .05 & $>.05$ & & & \\
\hline & Etiology & 16.99 & .33 & 2.75 & $<.01$ & & & \\
\hline \multirow[t]{3}{*}{ Full IQ } & Sex & -9.12 & -.21 & -1.69 & $>.05$ & \multirow[t]{3}{*}{.21} & \multirow[t]{3}{*}{5.01} & \multirow[t]{3}{*}{.01} \\
\hline & Age & .17 & .00 & .03 & $>.05$ & & & \\
\hline & Etiology & 15.03 & .36 & 2.97 & $<.01$ & & & \\
\hline
\end{tabular}

It can be observed in Table 2 that etiological variability $(\beta=.37 ; \mathrm{t}=2.99 ; \mathrm{p}<.05)$ was the only factor that independently predicted Verbal IQ among the epileptic children. This implies that children with idiopathic epilepsy reported higher Verbal IQ than children classified as symptomatic epilepsy. However, all the predictor variables (etiological variability,sex and age) jointly predicted Verbal IQ $(\mathrm{F}[3,56]=4.27 ; \mathrm{p}<.01)$ with $\mathrm{R}^{2}=$ 19. This suggests that all the predictor variables accounted for 19 percent of the proportion of variance in Verbal IQ. This means that sex, age and etiological variability are significant in understanding Verbal IQ.

Further, multiple regressions statistical analysis was used to test the independent and joint variables that would yield an optional predictive equation of Performance Intelligence Quotient (Performance IQ). The result revealed that $\operatorname{sex}(\beta=-.27 ; \mathrm{t}-2.13 ; \mathrm{p}<.05)$ and etiological variability $(\beta=.33 ; \mathrm{t}=2.75 ; \mathrm{p}<.05)$ independently predicted Performance IQ. This result indicates that the male epileptic children reported higher Performance IQ than the female epileptic children. Subsequently, children whose epilepsy could be traced to certain organic cause scored significantly less on Performance IQ than those whose epilepsy could not be traced to a known cause. Nevertheless, all the variables (etiological variability, sex and age) jointly predicted Performance IQ $(\mathrm{F}[3,56]=5.30 ;<.05)$ with $\mathrm{R}^{2}=22$. This suggests that all the predictor variables together accounted for 22 percent of the proportion of variance in Performance IQ.

Lastly, multiple regressions statistical analysis was used to test the independent and joint variables that would yield an optional predictive equation of Full Scale IQ. As shown in Table 2, etiological variability $(\beta=$ $.36 ; \mathrm{t}=2.97 ; \mathrm{p}<.05)$ was the only factor that independently predicted Full Scale IQ. This shows that the children with idiopathic epilepsy reported higher Full Scale IQ than those with symptomatic epilepsy. Etiological variability, sex and ageas predictor variables jointly predicted Full Scale IQ $(\mathrm{F}[3,56]=5.01 ; \mathrm{p} .05)$ with $\mathrm{R}^{2}=21$. This suggests that all the predictor variables accounted for 21 percent of the proportion of variance in Full Scale IQ. 


\section{Discussion}

Intellectual functioning in children with epilepsy can present different patterns and severity depending on numerous factors involved in this syndrome, such asetiological variability, sex and age. This study examined these epileptic factors as determinants of general intellectual functioning among the children. Based on the analysis, this study revealed that close to two third of the epileptic children examined were found to be experiencing developmental disability. Various factors are considered likely to induce cognitive disabilities in this population: the age of onset, period of time since onset, the type of epilepsy, the nature and frequency of the seizures, and the effects of antiepileptic treatment [24]. Elgar et al.[7] claim that neuropsychological impairment is an important co-morbidity of chronic epilepsy. Guzeva et al. [28] also assert that it is fairly common that children with epilepsy have educational problems and cognitive deficits.

The findings of this study also revealed that etiological variability independently predicted Verbal IQ, Performance IQ and Full IQ among epileptic children. This implies that children with idiopathic epilepsy reported higher Verbal IQ, Performance IQ and Full IQ than children classified as symptomatic epilepsy. The findings are in line with the assertion of Svoboda [21], who avers that those developmental problems are more common in children with symptomatic epilepsy than in children with idiopathic epilepsy.Takagi [18] also found that both the Verbal IQ and Performance IQ were significantly lower in the symptomatic group than in the idiopathic group. Van Rijckevorsel[16]also notes that some idiopathic focal epilepsy may have a favourable progression with a rapid and absolute seizure control and no cognitive deficits. Bourgeois et al. [15] also observe that children with symptomatic epilepsy are at a greater risk for cognitive impairment than are those with idiopathic epilepsy. Also, claim that most idiopathic, straightforward epilepsies cognition is mildly impaired or within normal range [22].

This study also shows that sex independently predicted Performance IQ of epileptic children. This result indicates that the male epileptic children reported higher Performance IQ than the female epileptic children. However, sex did not predict verbal IQ and Full Scale IQ in this study. The finding correlates with those of previous studies. For example, Sharma et al. [11] concluded that gender had no impact on neurological and psychological deficits among epileptic children. Other comparable results, such as Hoare and Kerley[29], found gender difference to be inconsistent as a predictor of psychological problems in children with epilepsy. Studies that found sex to predict cognitive functions have shown that male epileptic children had more neurological deficits than their female counterparts, but female epileptic children had higher mean psychological deficits.

Age did not significantly predict verbal IQ, Performance IQ and Full Scale IQ of epileptic children in this study. This result is contrary to the finding of Sharma et al. [11], who, in the assessment of the neuropsychological deficits among epileptic children,found that both neurological deficits and psychological deficits were highest in the age group of epileptic children who were between 13 and 16 years. They hinted that most epileptic children with neurological deficits had age at onset of seizures at 11-13 years but psychological deficits were more among epileptic childrenwith onset of seizure before the age of 4 years.

The findings of this study also emphasized a significant joint prediction of etiological variability, sex and age on Verbal IQ, Performance IQ and Full Scale IQ among the epileptic children. The results of the present study are supported by the findings ofDodrill[9], who established that many studies have described relationships involving cognitive status and a diversity of clinical epileptic features which include etiology, age of onset, seizure type and severity, duration, antiepileptic medications, and other factors [8].

\section{Conclusion}

This study found that close to two third of the epileptic children were experiencing developmental disability. An independent examination of the variables showed that etiological variability independently predicted Verbal IQ, Performance IQ and Full IQ among the epileptic children. Sex independently predicted Performance IQ of epileptic children. Furthermore, this study found a significant joint prediction of etiological variability, sex and ageon Verbal IQ, Performance IQ and Full Scale IQ among the epileptic children.Some features of the present study limit the conclusions that can be drawn and suggest directions for future research. The effects of other epilepsy-related factors, such as emotional factors, personality development, environmental factors, parental attitudes, concomitant handicaps and neurophysiologic impairment, were not included as part of the variables of investigation. Wechsler Intelligence Scale for Children was the only instrument used to assess intellectual functions among epileptic children. Other batteries of test, such as Wechsler Pre-School and Primary Scale and Raven's Standard Progressive Matrices, would also have been relevant in this study. 


\section{References}

[1]. K. Rantanen, Neurocognitive functioning of preschool children with 'Epilepsy only'Lisensiaatintutkimus, 19 s., 4 liitesivua, 2006

[2]. V. Anderson, E. Northam, J. Hendy, and J.Wrennall, Developmental neuropsychology. A clinical approach. 2001, Hove: Psychology Press.

[3]. V. I. Guzeva, Epilepsy and Non-Epilepstic Convulsive States in Children (in Russian), MIA, Mosco: 2007, pp. 327-335

[4]. W. Hauser, J. Annegers, and W. Rocca, Descriptive epidemiology of epilepsy: Contributions of population-based studies from Rochester, Minnesota. Mayo Clinic Procedings , 71 (6), 1996, 576-586.

[5]. W. S. MacAllister, and S. G. Schaffer, Neuropsychological deficits in childhood epilepsy syndromes. Neuropsychology Review, 17, 2007, 427-444.

[6]. M. Westerveld, Childhood Epilepsy. In K. O. Yeates, D. M. Ris, H. G. Taylor, and B. F. Pennington (Eds.), Pediatric Neuropsychology: Research, Theory, and Practice (New York: The Guilford Press, 2010) 71-91

[7]. C. E. Elger, C. Helmstaedter, and M. Kurthen, Chronic epilepsy and cognition. Lancet Neurology, 3, $2004,663-672$.

[8]. A. P. Aldenkamp, and Arends, J. Effects of epileptiform EEG discharges on cognitive function: is the concept of "transient cognitive impairment" still valid? Epilepsy and Behaviour, 5, (2004), S25-S34.

[9]. C. B. Dodrill, Neuropsychological effects of seizures.Epilepsy \& Behaviour, 5, 2004, S21-S24

[10]. S. E. Sabbagh, C. Soria, S. Escolano, C. Bulteau, and G. Dellatolas, Impact of Epilepsy Characteristics and Behavioral Problems on School Placement in Children. Epilepsy \& Behaviour, 9, 2006, 573-578.

[11]. A. Sharma, P. Singh, S. Goval, M. Singh, and H. Kaur, A Descriptive study to assess the neuropsychological deficits among epileptic children. Delhi Psychiatry Journal, 14 (14), 2011, 78-87.

[12]. S. L. Rao, Cognitive deficit in epilepsy. National Institute of Mental Health and Neuro Sciences Journal, 11(1), 1993, 11-19.

[13]. S. J. You, Cognitive function of idiopathic childhood epilepsy. Korean Journal of Pediatrics, 55 (5),2012, $159-163$.

[14]. V. V. Bhise, G. D. Burack, and D. E. Mandelbaum, Baseline cognition, behavior, and motor skills in children with new-onset idiopathic epilepsy. Developmental Medicine \& Child Neurology, 52, 2010, 22-26.

[15]. B. F. Bourgeois, A. L. Prensky, H. S. Palkes, B. K. Talent, and S. G. Busch, Intelligence in epilepsy: a prospective study in children. Annals of Neurology, 14, 1983, 438-444.

[16]. A. T. Berg, S. N. Smith, D. Frobish, B. Beckerman, S. R. Levy, F. M. Testa, et al. Longitudinal assessment of adaptive behavior in infants and young children with newly diagnosed epilepsy: influences of etiology, syndrome, and seizure control.Pediatrics, 114, 2004, 645-650.

[17]. K. Van Rijckevorsel, Cognitive problems related to epilepsy syndromes, especially malignant epilepsies. European Journal of Epilepsy, 15 (4), 2006, 227-234.

[18]. A. T. Berg, S. N. Smith, D. Frobish, B. Beckerman, S. R. Levy, F. M. Testa, et al. Longitudinal assessment of adaptive behavior in infants and young children with newly diagnosed epilepsy: influences of etiology, syndrome, and seizure control.Pediatrics, 114, 2004, 645-650.

[19]. K. Takagi, Comprehensive neuropsychological analysis of epileptic children with WISC-R, including profile analysis of subjects. No to Hattatsu, 28 (1), 1996, 30-38.

[20]. C. Hommet, C. Billard, J. Motte, G. D. Passage, D. Perrier, P. Gillet, et al., Cognitive function in adolescents and young adults in complete remission from benign childhood epilepsy with centro-temporal spikes. Epileptic Disorders, 3, $2001,207-216$.

[21]. E. Rodin, Prognosis of cognitive functions in children with epilepsy. In B. P. Hermann and M. Seidenberg (Eds.), Childhood epilepsies: Neuropsychological, psychosocial and intervention aspects (Chichester: John Wiley \& Sons. 1989) 33-50

[22]. W. B. Svoboda, Childhood epilepsy. Language, learning, and abnormal behavioural complications (Cambridge: Cambridge University Press, 2004)

[23]. C. Hommet, H. C. Sauerwein, B. De Toffol, and M. Lassonde, Idiopathic epilepsy syndromes and cognition. Neuroscience \&Biobehavioural Reviews, 30, 2006, 85-96

[24]. G. Motamedi, and K. Meador, Epilepsy and cognition. Epilepsy \& Behaviour, 4, 2003, S25-S38.

[25]. A. P. Aldenkamp, J. Overweg, and L. A. M. Diepman, Factors involved in learning problems and educational delay in children with epilepsy. Child Neuropsychology, 5, 1999, 130-136.

[26]. Y. Henkin, M. Sadeh, S. Kivity, E. Shabtai, L. Kishon-Rabin, and N. Gadoth, Cognitive function in idiopathic generalized epilepsy of childhood. Developmental Medicine \& Child Neurology, 47, 2005, 126-132.

[27]. T. Deonna, P. Zesiger, V. Davidoff, M. Maeder, C. Mayor, and E. Roulet, Benign partial epilepsy of childhood: a longitudinal neuropsychological and EEG study of cognitive function. Developmental Medicine \& Child Neurology, 42, $2000,595-603$.

[28]. E. Germano, A. Gagliano, A. Magazu, C. Sferro, T. Calarese, E. Mannarino, et al., Benign childhood epilepsy with occipital paroxysms: neuropsychological findings. Epilepsy Research, 64, 2005, 137-150.

[29]. V. I. Guzeva, V. O. Belash, V. V. Guzeva, O.V. Guzeva, and O.I. E. Anastazi, Characteristics of cognitive functions in children with epilepsy. Neuroscience and Behavioural Physiology, 39 (9), 2009, 885-889.

[30]. P. Hoare, and S. Kerley, Psychosocial adjustment ofchildren with chronic epilepsy. Neurology, 33, 1991, $3001-3215$.

[31]. J. Austin, J. Harezlak, D. Dunn, G. Huster, D. Rose, and W. Ambrosius, Behavior problems in children before first recognized seizures. Pediatrics, 107 (1), 2001, 115-122.

[32]. R. Sridharan, and B. N. Murthy, Prevalence and pattern of epilepsy in India. Apollo Hospitals, Institute for Research in Medical Statistics, Indian Council of Medical Research, Chennai, Madras. Epilepsia,40 (5),2007, 631-636. 